\title{
Facetas diretas em dentes anteriores utilizando composite wetting resin
}

\author{
Direct veneers on anterior teeth using composite wetting resin \\ Facetas directas en dientes anteriores con resina compuesta humectante
}

Recebido: 15/09/2021 | Revisado: 20/09/2021 | Aceito: 21/09/2021 | Publicado: 23/09/2021

Vinícius Andrade Ribeiro Peres

ORCID: https://orcid.org/0000-0002-2304-5047

Faculdade Patos de Minas, Brasil

E-mail: sonvinicius7@gmail.com

Ester Marieta da Silva

ORCID: https://orcid.org/0000-0002-8016-1271 Faculdade Patos de Minas, Brasil

E-mail: estermarieta123@gmail.com

Juliana Franco Monteiro

ORCID: https://orcid.org/0000-0003-3091-9459

Universidade Federal de Uberlândia, Brasil

E-mail: julianafrancomont@gmail.com

Lia Dietrich

ORCID: https://orcid.org/0000-0001-7887-8591 Universidade Federal do Vale do Jequitinhona e Mucuri, Brasil

E-mail: lia_dietrich@yahoo.com.br

Cláudia Maria de Oliveira Andrade

ORCID: https://orcid.org/0000-0003-4529-8106 Faculdade Patos de Minas, Brasil

E-mail: Claudiamoacd@yahoo.com.br

Murilo Guimarães Campolina

ORCID: https://orcid.org/0000-0002-6079-8718

Universidade Federal de Uberlândia, Brasil E-mail: gc.murilo.gc@gmail.com

Victor da Mota Martins

ORCID: https://orcid.org/0000-0001-6631-6161

Centro Universitário de Patos de Minas, Brasil

E-mail: victortag@hotmail.com

\begin{abstract}
Resumo
Introdução: Diastemas estão entre as principais queixas estéticas dos pacientes e podem ser classificados como espaços extras, localizados entre dois dentes ou mais, causados por diferenças de tamanhos dentais, ausência dos mesmos, desproporção dos dentes no arco superior com inferior, hábitos deletérios e vários outros fatores. A busca por tratamentos conservadores possibilitou o desenvolvimento de resinas cada vez mais aprimoradas, que visam o estabelecimento de restaurações com estética satisfatória em um menor tempo de trabalho, sem necessidade de maiores desgastes, preservando a estrutura dental. Objetivos: Relatar um caso clínico onde foi restabelecida a estética em diastemas anteriores com o uso de resinas diretas e agentes umectantes, evitando maiores desgastes, devolvendo função e autoestima para o paciente. Descrição do caso: Foi realizado protocolo fotográfico, clareamento de consultório e caseiro, seguido de enceramento diagnóstico, com montagem em articulador, verificação de guias, confecção de matriz de silicone para obter a incisal, seguida do fechamento dos diastemas com resinas diretas e uma fina camada superficial com agente umectante. Considerações Finais: A resina composta se mostrou uma excelente alternativa quando utilizada de maneira correta por um profissional capacitado, que compreenda os princípios estéticos e psicológicos de seu paciente. A utilização de agente umectante associado a resina composta proporcionou o sucesso do tratamento restaurador facilitando manipulação da resina e consequentemente a anatomia, superando as expectativas do paciente, devolvendo ao mesmo a estética de seus dentes, elevando, assim, sua autoestima.
\end{abstract}

Palavras-chave: Diastema; Estética dentária; Resina composta.

\begin{abstract}
Introduction: Diastemas are found among the main aesthetic ones of patients and can be classified as extra spaces, located between the most important ones, caused by differences in the size of the two faces, absence of differences, disproportion of faces in the upper arch and lower, deleterious habits and several others. factors. The search for conservative treatments allowed the development of increasingly improved resins, which aim to establish restorations with satisfactory aesthetics in a shorter time of work, without the need for greater wear, preserving the dental structure. Objectives: To present a clinical case where aesthetics can be reestablished in previous diastema with the use of direct resins and wetting agents, avoiding further wear, restoring function and self-esteem to the patient. Description of the
\end{abstract}


case: A photographic protocol was carried out, whitening in the office and at home, followed by a diagnostic wax-up, assembly in an articulator, revision of guides, elaboration of a silicone matrix to obtain the incisal, followed by a circle of the diastema with direct resins and a thin surface layer with a wetting agent. Final considerations: The composite resin has proven to be an excellent alternative when it correctly uses a trained professional who understands the aesthetic and psychological principles of your patient. The use of a humectant agent associated with composite resin provided the success of the restorative treatment, facilitating the manipulation of the resin and, consequently, the anatomy, surpassing the patient's expectations, restoring the aesthetics of their clients, thus increasing their self-esteem.

Keywords: Diastema; Dental esthetics; Composite resin.

\begin{abstract}
Resumen
Introducción: Los diastemas se encuentran entre las principales quejas estéticas de los pacientes y se pueden clasificar como espacios extra, localizados entre dos o más dientes, provocados por diferencias en el tamaño de los dientes, ausencia de los mismos, desproporción de los dientes en la arcada superior e inferior, hábitos deletéreos y varios otros. factores. La búsqueda de tratamientos conservadores permitió el desarrollo de resinas cada vez más mejoradas, que tienen como objetivo establecer restauraciones con una estética satisfactoria en un tiempo de trabajo más corto, sin necesidad de un mayor desgaste, preservando la estructura dental. Objetivos: Presentar un caso clínico donde se restableció la estética en diastemas previos con el uso de resinas directas y agentes humectantes, evitando un mayor desgaste, devolviendo la función y la autoestima al paciente. Descripción del caso: Se realizó un protocolo fotográfico, blanqueamiento en consultorio y en casa, seguido de encerado diagnóstico, montaje en articulador, revisión de guías, elaboración de una matriz de silicona para obtener el incisal, seguido de cierre del diastema con resinas directas y una fina capa superficial con agente humectante. Consideraciones finales: La resina compuesta demostró ser una excelente alternativa cuando la utiliza correctamente un profesional capacitado que comprende los principios estéticos y psicológicos de su paciente. El uso de un agente humectante asociado a resina compuesta proporcionó el éxito del tratamiento restaurador, facilitando la manipulación de la resina y, en consecuencia, la anatomía, superando las expectativas del paciente, devolviendo la estética de sus dientes, aumentando así su autoestima.
\end{abstract}

Palabras clave: Diastema; Estética dental; Resina compuesta.

\title{
1. Introdução
}

Tratamentos restauradores estéticos são altamente procurados na odontologia atualmente, já que a exigência à adesão aos padrões vigentes é cada vez maior. Grande parte dos pacientes que entram em um consultório odontológico anseiam por dentes harmônicos e bem posicionados (Mondelli, 2017), uma vez que um sorriso bem estruturado, com estética satisfatória e proporções corretas possibilita o desenvolvimento de maior autoconfiança, que está diretamente a fatores mentais, maior qualidade de vida e autoestima (Marques, 2019). Nesse contexto, é de suma importância o oferecimento de tratamento restaurador adequado e de qualidade, que proporcione o bem-estar e a inclusão social (Oliveira, et al., 2020).

Como preocupação estética primária enquadram-se os diastemas (Chu Ch, et al., 2011; Romero, et al., 2018), que podem ser classificados como espaços extra localizados entre dois dentes ou mais, causados por diferenças de tamanhos dentais, ausência dos mesmos, desproporção dos dentes no arco superior com inferior, hábitos deletérios e vários outros fatores (Canuto, et al., 2006). Normalmente é considerado como diastema espaços interdentais maiores que $0,5 \mathrm{~mm}$ mais comumente localizados entre incisivos centrais superiores (Keene, 1963). Quando definida sua etiologia, diversos tratamentos podem ser ofertados, como cirurgia, ortodontia, procedimentos restauradores ou uma associação entre eles (Romero, 2018).

Dadas as inúmeras opções de tratamento, as decisões de fechamento de diastemas são fundamentadas em uma análise de cada caso e em princípios como economia, disponibilidade de tempo e desejo do paciente (De Campos, 2015). Além disso, um dos grandes fatores de influência na escolha tem sido a busca por terapêuticas conservadoras, já que com o desenvolvimento da tecnologia adesiva e de resinas cada vez mais aprimoradas (Michelon, et al., 2009), tornou-se possível acrescentar compósitos às estruturas dentárias com pouco ou nenhum preparo cavitário, de modo minimamente ou não invasivo (Lempel, et al., 2017; Ferracane, 2011; Frese, et al., 2013).

Como consequência do aprimoramento das resinas compostas a realização de tratamentos estéticos com as mesmas se elevou consideravelmente. $\mathrm{O}$ avanço em suas fórmulas gerou resinas capazes de restaurar adequadamente dentes anteriores e posteriores tendo como base princípios estéticos, biológicos e funcionais (Schwarz, et al., 2013). Além disso, esses compósitos 
são materiais que podem ser usados para tratamento restaurador em sessão única. No entanto, a principal desvantagem está correlacionada à estabilidade da cor e possíveis fraturas da resina, apesar de diferentes estudos demonstrarem uma alta taxa de sucesso de 5 a 10 anos (Lempel, et al., 2017; Frese, et al., 2013).

O fechamento de um grande diastema com resina composta apresenta alguns desafios: o de fechá-lo sem que a largura dos dentes fiquem desproporcionais e sem que haja excessos de compósitos nas proximais, o que seria um fator predisponente para o acúmulo de alimentos e posteriormente, para o desenvolvimento de cálculos (Calamia,2015), além da dificuldade de inserção e manipulação do material na cavidade dental. Considerando essas dificuldades, o presente caso clínico descreve um enceramento diagnóstico prévio realizado para que as proporções de largura pudessem ser adequadamente realizadas e analisadas (Calixto, et al., 2011). Além disso, o procedimento conta com a utilização de um modelador de resina para posicionar, manipular e esculpir de modo mais facilitado e aprimorado todas as restaurações diretas em compósito, melhorando a modelagem do material e a adaptação do mesmo à cavidade dentária (Lynch, 2011).

O modelador de resinas vem sendo empregado para melhorar o manuseio clínico das resinas compostas, já que grande parte desses compósitos presentes no mercado odontológico atual possuem aderência às espátulas de inserção, o que dificulta a confecção da anatomia dental tão crucial ao procedimento restaurador, podendo gerar falhas no interior da restauração. (Bayraktar, 2021). Formações de bolhas ou defeitos coesivos da resina estão entre as principais consequências e podem afetar diretamente as propriedades ópticas da restauração, sobretudo a translucidez e a estabilidade de cor (Munchow, 2016).

Assim, este trabalho tem como objetivo o relato de um caso clínico de restabelecimento da estética nos diastemas com a fabricação de facetas diretas utilizando agente modelador de resina. Foi proposto o uso de resinas diretas, as quais o desgaste dentário foi evitado, para reconstrução dos espaços interdentais e reestruturação da proporção dental, devolvendo função, estética e autoestima ao paciente.

\section{Metodologia}

Este artigo é um relato de caso descritivo, com foco qualitativo e exploratório. Aborda, como tema principal, a utilização clínica de agente umectante para manuseio de resina composta utilizada no procedimento odontológico de fechamento de diastemas. Leva em conta a experiência dos investigadores na utilização do produto (composite wetting resin) e descreve, com embasamento na literatura, todas etapas utilizadas no procedimento clínico abordado sobre fechamento de diastema (Estrela, 2018).

Utiliza de um método qualitativo, uma vez que permite o estudo em profundidade de uma temática específica (Vazquez, et al., 2015), analisando-a de forma estrutural, teoricamente fundamentada e interpretada. Parte do próprio contexto e aborda questões que despertam a autopercepção e autocrítica.

\section{Aprovação do Comitê de Ética e Pesquisa}

Sabe-se que a ética vai muito além do âmbito científico e é a responsável pelo norteamento da conduta de um determinado procedimento ou de uma determinada pessoa. Justamente por isso, faz-se necessária a abordagem do posicionamento do paciente frente ao tratamento proposto.

Isto posto, o paciente do presente artigo concordou em participar deste trabalho e assinou o Termo de Consentimento Livre e Esclarecido, sendo o projeto tramitado pelo Comitê de Ética e Pesquisa com Seres Humanos da Faculdade Patos de Minas, autorizado pelo número de parecer: 4.683.415. 


\section{Descrição do Caso}

Paciente V.P., gênero masculino, 22 anos, chegou à policlínica da Faculdade Patos de Minas - FPM, se queixando de diastemas em seus dentes ântero superiores, com desejo de restabelecer a harmonia dental. Após anamnese e exame clínico, foi estabelecida a abordagem de tratamento para correção estética dos dentes 11, 12, 21 e 22, onde os diastemas se mostravam presentes entre os incisivos centrais e laterais de ambas as hemiarcadas, e possuíam tamanho insatisfatório, gerando incômodo visual relatado pelo paciente (Figura 1).

O tratamento iniciou-se com profilaxia com pedra-pomes e água, seguida por clareamento dentário de consultório, feito em 3 sessões de 15 minutos (intervalo de 7 dias entre cada sessão). Foram necessárias 3 sessões de clareamento devido a saturação do substrato dentário. Os materiais utilizados foram gel clareador peróxido de hidrogênio a 35\% (Whiteness HP - FGM. Joinville, SC, Brasil) e barreira gengival (Top Dam - FGM. Joinville, SC, Brasil) para proteção das estruturas adjacentes. Além do clareamento de consultório, também foi feita moldagem da arcada superior e inferior do paciente com alginato (Hydrogum Zhermack S.p.a., Badia Polesine, Rovigo, Itália) para confecção de um modelo de estudo e fabricação de uma placa para clareamento caseiro, realizado com peróxido de carbamida 16\%, uso noturno por 21 dias, garantindo a longevidade do tratamento clareador. O modelo de estudo também foi utilizado em articulador semi ajustável para a obtenção de um enceramento diagnóstico e de uma matriz de silicone pelo laboratório de prótese dentária (Figura 2).

Obtendo uma prévia do resultado do procedimento restaurador, sugeriu-se ainda mais acréscimos em resina em incisivos centrais e laterais para a promoção de alinhamento estético com caninos. Houve discordância por parte do paciente, que desejava realizar uma alteração estética mais conservadora, mantendo a forma de seus dentes com estética semelhante à de origem. Logo, os demais acréscimos sugeridos não foram realizados. Passados 15 dias após a realização do clareamento dentário, deu-se início ao protocolo fotográfico inicial (Figura 1) e ao procedimento restaurador.

Figura 1 - A,B- Imagens do paciente em posição frontal sorrindo e com a boca relaxada/ semiaberta, respectivamente; C,DImagens focadas no sorriso e posição de boca relaxada/ semiaberta do mesmo; E,F- Imagens de perfil do sorriso, e boca relaxada/ semiaberta, mostrando a posição vestibularizada dos dentes no arco.

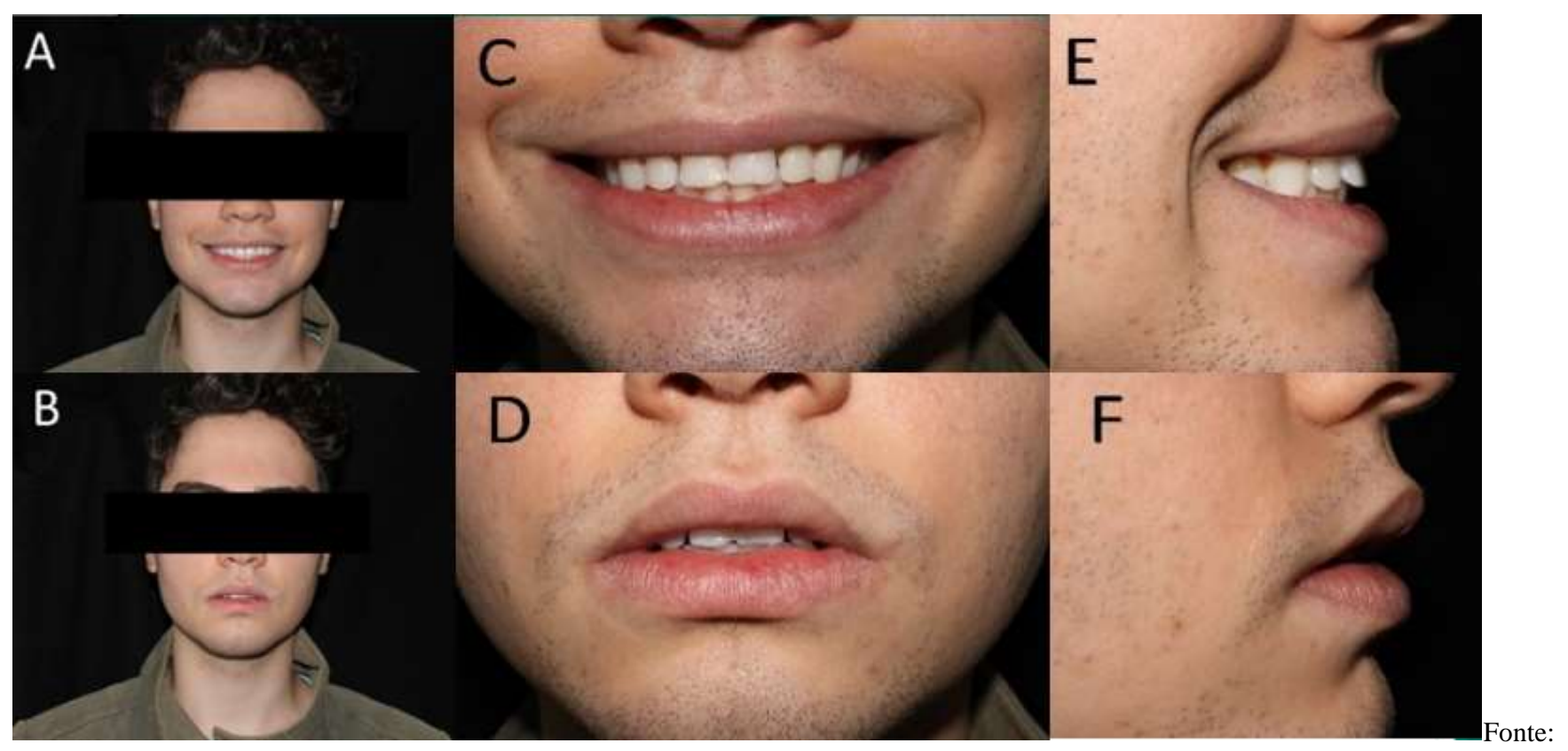

Imagens autorais (2021) 
Figura 2 - A- Montagem de modelo superior e inferior no Asa (Articulador Semi Ajustável), e enceramento diagnóstico dos dentes 11,12, 21,22; B- Imagem de modelo superior, com a adaptação da matriz de silicone; C- Imagem da matriz de silicone finalizada.

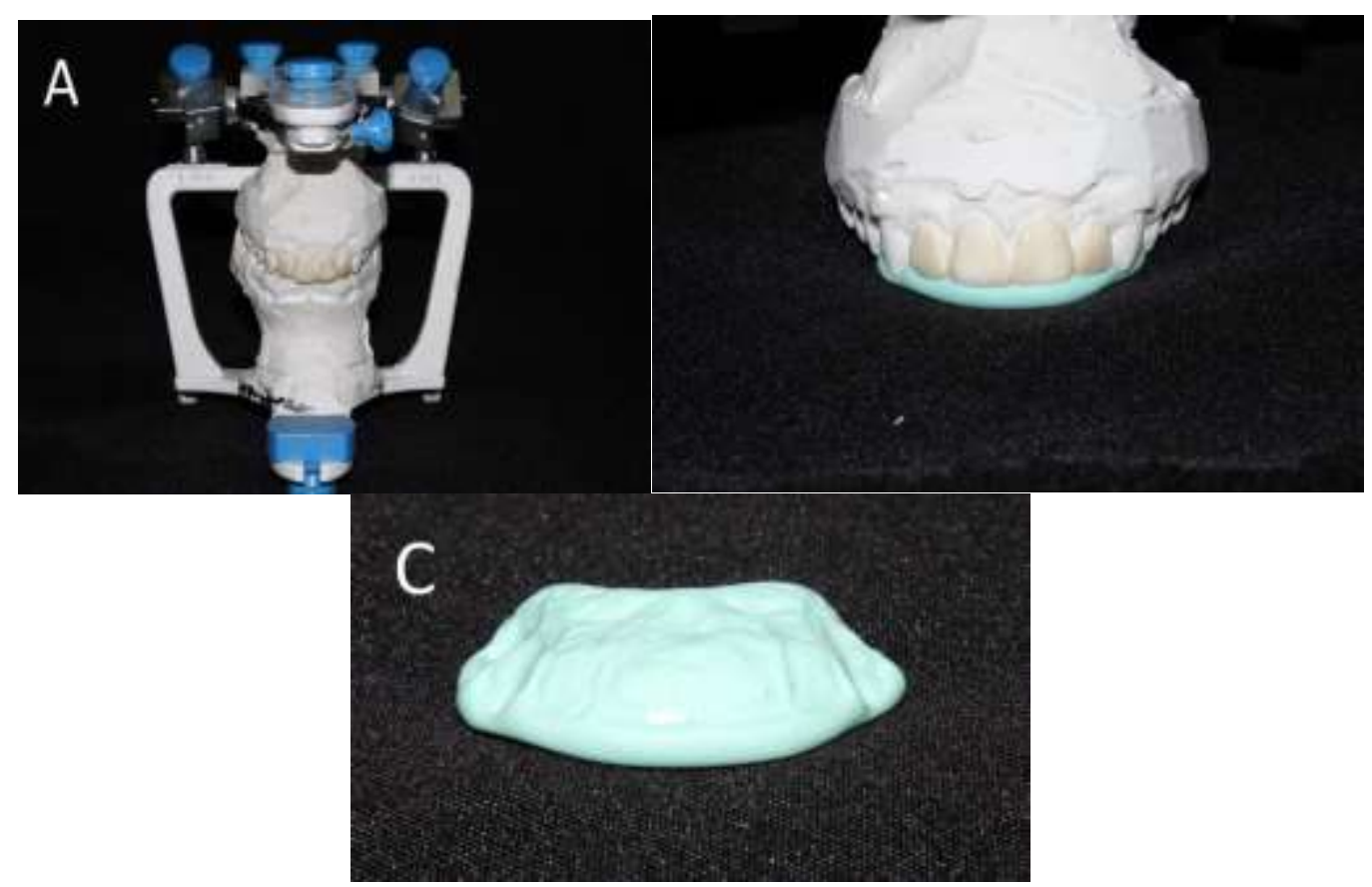

Fonte: Imagens autorais (2021).

Primeiramente foi realizada a limpeza dos dentes com escova de Robinson e pedra pomes diluída em água para remoção de quaisquer impurezas presentes. Em seguida realizou-se a análise da coloração com escala Vita, dando segmento ao isolamento absoluto modificado com inserção do fio retrator (Retraflex - Biodinâmica) para melhor visualização do término cervical, sem necessidade de anestesia local. Com a região de trabalho isolada e bem delimitada, iniciou-se o processo de condicionamento do esmalte dental utilizando ácido fosfórico 37\% (Maquira, Maringá, PR, Brasil) somente nos incisivos centrais superiores durante 30 segundos, seguido de irrigação com água pelo mesmo tempo de aplicação do ácido e secagem com papel absorvente para controle de umidade nos dentes. Aplicou-se adesivo convencional de dois passos com primer e bond no mesmo frasco (Adper Single Bond 2 - 3M/ESPE, St. Paul, MN, EUA), espalhando-o por 15 segundos na superfície dental por duas vezes seguidas, para então realizar a fotopolimerização. Com o adesivo fotopolimerizado, iniciou-se a inserção de resina translúcida (Filtek CT Z350 XT - 3M/ESPE, St. Paul, MN, EUA) na matriz de silicone para confeccionar a face palatina e incisal dos dentes 11 e 21 , fotoativadas (GNATUS - $1200 \mathrm{~mW} / \mathrm{cm}^{2} \pm 200 \mathrm{~mW} / \mathrm{cm}^{2}$ ), por tempo padrão de 20 segundos cada. Incrementos na mesial e distal dos dentes foram feitos, para o fechamento dos diastemas, com o auxílio de uma matriz (Unimatrix - TDV. Pomerode, SC, Brasil) para facilitar a formação das proximais. Novamente os incrementos foram fotoativados (20 segundos) e seguiu-se para os incrementos da resina de esmalte (Filtek A1E e A1B Z350 XT - 3M/ESPE, St. Paul, MN, EUA), também fotopolimerizados. O último incremento foi feito na face vestibular, aliado ao modelador de resina (BISCO, Illinois, Schaumburg). O modelador foi dispensado em um pote dappen no qual o pincel pelo marta (Tigre, Joinville, SC, Brasil) foi inserido para devida embebição e posterior aplicação. O excesso do modelador foi removido em gaze e o pincel embebido com o modelador foi aplicado sobre a última camada da restauração, posteriormente também fotoativada. Terminadas as restaurações dos dentes 11 e 21 , deu-se início a realização do procedimento nos incisivos laterais (12 e 22), feito em padrão semelhante ao condicionamento ácido, controle de umidade, aplicação de adesivo e técnica restauradora dos incisivos centrais já realizados. Criou-se a face palatina dos dentes com resina translúcida, que recebeu fotoativação por 20 segundos, seguido das paredes proximais em resina com auxílio da matriz (Unimatrix - TDV. Pomerode, SC, Brasil), posteriormente fotoativada. Os incrementos de esmalte foram realizados com o 
auxílio do agente umectante e fotoativados e, finalizando, houve aplicação de gel hidrossolúvel na vestibular, fotoativada pela última vez (Figura 3).

Figura 3 - A- Execução do isolamento absoluto modificado; B- Inserção do fio retrator (Retraflex - Biodinâmica) sem necessidade de anestesia local; C- Condicionamento do esmalte dental (11 e 21) utilizando ácido fosfórico 37\% (Maquira, Maringá, PR, Brasil) por 30 segundos; DIrrigação por 30 segundos e secagem; E- Aplicação de adesivo convencional de dois passos (no 11 e 21) com primer e bond no mesmo frasco (Adper Single Bond 2 - 3M/ESPE, St. Paul, MN, EUA) por 15 s por duas vezes seguidas; F- Fotopolimerização; G- Inserção de resina translúcida (Filtek CT Z350 XT - 3M/ESPE, St. Paul, MN, EUA)na matriz de silicon (11 e 21) fotoativada por 20 segundos cada face; fez-se incrementos na mesial e distal dos dentes para o fechamento dos diastemas, com o auxílio de uma matriz (Unimatrix - TDV. Pomerode, SC, Brasil); H-Incremento da resina de esmalte (Filtek A1E e A1B Z350 XT - 3M/ESPE, St. Paul, MN, EUA) no 11 e 21- fotoativação; I- O último incremento foi feito na face vestibular aliado ao modelador de resina (BISCO, Illinois, Schaumburg). O mesmo processo foi repetido para os laterais.

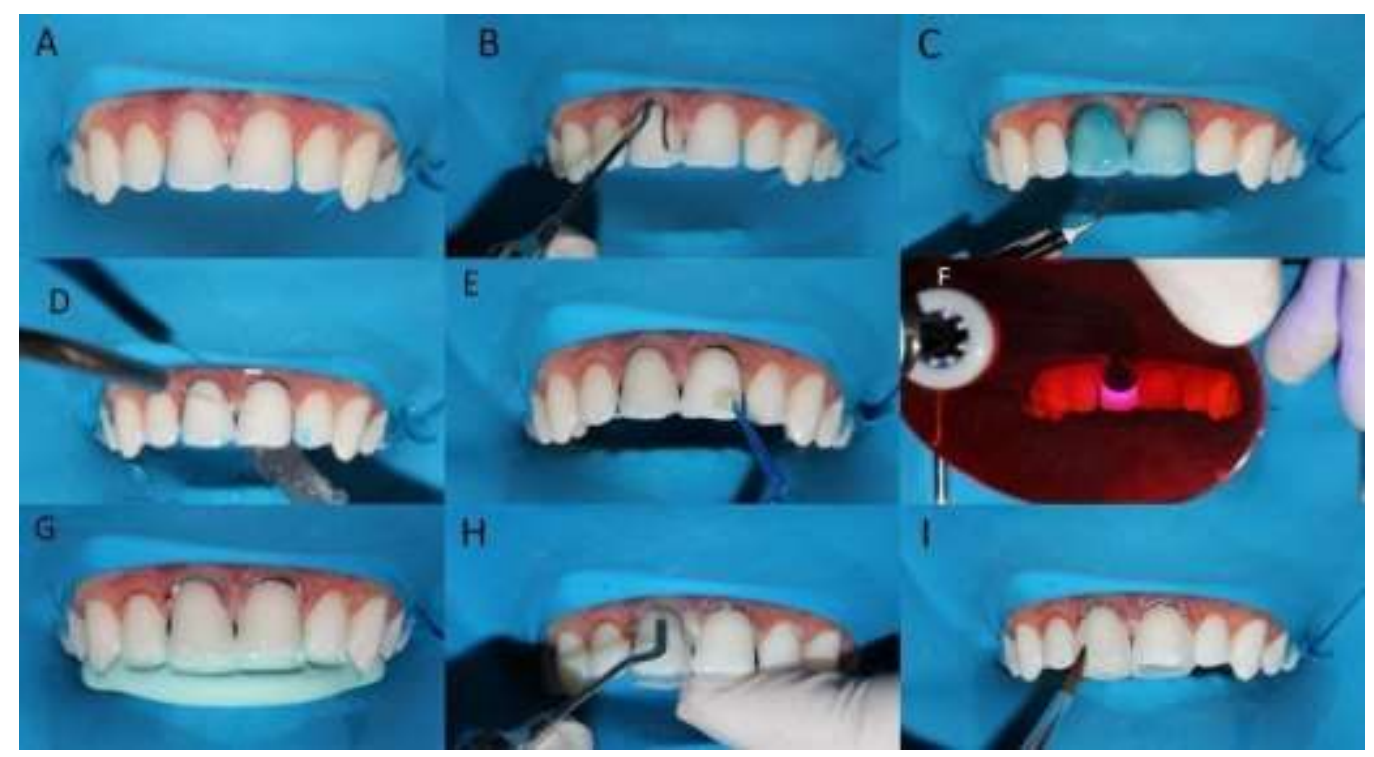

Fonte: Imagens autorais (2021).

Terminado o procedimento restaurador, a oclusão do paciente foi chegada com papel carbono para remoção de quaisquer contatos excessivos presentes, seguido de acabamento com as brocas diamantadas F e FF (KG Sorensen. Cotia, SP, Brasil) para as faces vestibular e palatina dos dentes restaurados. Tiras de lixa de resina foram utilizadas nas faces mesial e distal, removendo assim as irregularidades que promoveriam acúmulo de resíduos. Foi feito polimento para aumento do brilho e melhoria na textura com taças de borracha e um kit de polimento (Dhpro. Paranaguá, PA, Brasil). O retorno do paciente foi marcado para o dia seguinte.

\section{Resultados}

Paciente retornou 24 horas após finalização das facetas para finalização do acabamento e polimento queixando de cortes no lábio devido ao aumento dos dentes, foi realizado ajuste na altura dos elementos 11, 12, 21 e 22 e um pequeno desgaste a mais no dente 21, individualizado e de acordo com o desejo do paciente. O indivíduo se mostrou extremamente satisfeito com o resultado obtido e foi dada continuidade ao protocolo fotográfico final (Figura 4). 
Figura 4 - A,B- Imagens do paciente em posição frontal sorrindo e com a boca relaxada/ semiaberta, respectivamente após fechamento de diastemas e restaurações em resina composta; C,D- Paciente sorrindo e paciente com posição de boca relaxada/ semiaberta; E,F- Imagens de perfil do sorriso, e boca relaxada/ semiaberta, mostrando reabilitação estética e diminuição de overbite.

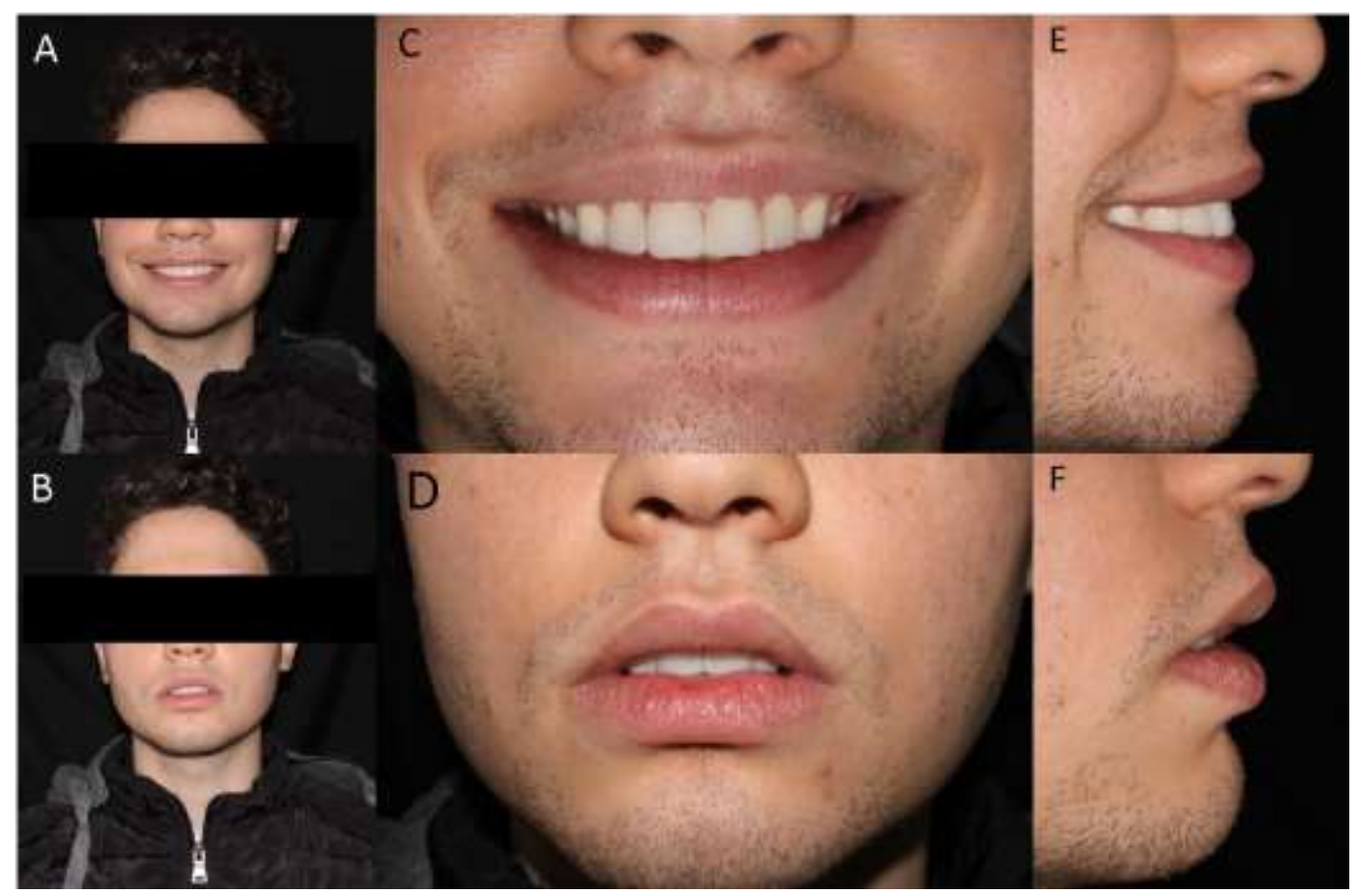

Fonte: Imagens autorais (2021).

\section{Discussão}

O papel do Cirurgião-Dentista, ao promover tratamentos estéticos dentários, se dá por meio da compreensão das necessidades do paciente, o que engloba a averiguação de opções de tratamento e das vantagens e desvantagens de cada protocolo clínico. É necessário realizar o plano de tratamento baseado nas singularidades, desejos e percepções de harmonia e beleza de cada indivíduo (Arndt, et al., 1986; Ahmad, 2005).

Nessa perspectiva, o paciente do presente trabalho possuía diastemas entre os dentes anteriores e desejava tratamento estético minimamente invasivo, visando melhora de sua autoestima. Para que fosse realizado o procedimento, uma série de etapas foram elencadas e criteriosamente seguidas. Entre elas, cita-se a escolha da forma de tratamento com resinas diretas, o clareamento dental antecedendo o procedimento restaurador, a eleição de agentes modeladores de compósitos, enceramento diagnóstico, isolamento absoluto, seleção de sistema adesivo, escolha de cor e manuseio das resinas compostas, acabamento e polimento.

Para realizar uma melhor análise e planejamento da forma dos dentes, o enceramento diagnóstico é de grande importância. Através dele é possível a obtenção de uma prévia da anatomia dental a ser posteriormente realizada no paciente (Lima \& Carnevale Filho, 2019; Calixto, et al., 2011). Nesse viés, a escultura finalizada no modelo de gesso possibilitou ao paciente do presente trabalho ter uma visão da forma que seus dentes iriam adquirir, além de permitir a criação de uma matriz guia de silicone, que facilitou a execução da restauração e possibilitou ao profissional uma melhor confecção do serviço devido a previsibilidade gerada.

O clareamento dental, proposto como etapa prévia às facetas diretas, surgiu no ano de 1989 (Barbosa, et al., 2017), e é um quesito indispensável quando se trata de fechamento de diastemas com resinas compostas, sendo indicado na grande maioria dos casos por não ser invasivo e contribuir de forma significativa na estética do sorriso. É baseado na utilização de agentes 
clareadores, de preferência oxidantes, como peróxido de hidrogênio ou peróxido de carbamida (Felix, et al, 2019), os quais atuam de forma a eliminar os agentes descolorantes localizados na estrutura dentária (Román- Rodríguez, et al., 2020).

Os agentes clareadores se dividem em dois grandes grupos: sistema de clareamento em consultório e em casa. Os clareadores em consultório são realizados por profissionais de saúde bucal em seus respectivos ambientes de trabalho e se caracterizam pelo uso de agentes oxidantes de elevadas concentrações, normalmente de $25 \%$ a 35\% de peróxido de hidrogênio (Mounika, et al., 2018) em um reduzido espaço de tempo (Sundfeld, et al., 2018). Devido a essas altas concentrações (RománRodriguez, et al., 2020), deve ser feito um isolamento dos tecidos moles com barreira gengival (Da Costa, et al., 2010) ou com um produto de barreira para pintura gengival de modo a evitar a ocorrência de possível irritabilidade da mucosa bucal.

O outro grande grupo de sistemas de clareamento são aqueles realizados em domicílio e podem ser subdivididos em duas categorias: produtos de clareamento dentário supervisionados profissionalmente e OTC. Os supervisionados profissionalmente consistem em clareamentos supervisionados pelo Cirurgião- Dentista e que tem como princípios a utilização de moldeiras de plástico, obtidas a partir da moldagem da arcada dentária do paciente, as quais serão preenchidas com gel clareador para entrar em contato com os dentes (Karadas \& Duymus, 2015). As concentrações do agente oxidante são inferiores às dos tratamentos em consultório, usualmente utilizando $5 \%$ a $22 \%$ para o peróxido de carbamida e $6 \%$ a $15 \%$ para o peróxido de hidrogênio (Rodríguez-Martínez, et al., 2019). Já os sistemas OTC são geralmente realizados sem a supervisão do dentista e englobam pastas clareadoras, géis especiais, fitas e até mesmo kits de sistemas de clareamento, a preços mais acessíveis, embora com confiabilidade e resultados duvidosos (Perozzo, et. al., 2017).

Vale ressaltar que as vantagens do clareamento em consultório abrangem a necessidade mínima de colaboração do paciente e resultados visíveis imediatos (Tay, et al.,2009; Kossatz et al., 2011), embora necessitem de outras aplicações para atingir um clareamento ideal do substrato dental (Haywood , 2006). Entretanto, apresentam como desvantagens o custo elevado, o aparecimento de possíveis irritabilidades na mucosa e hipersensibilidade dentinária associada ao tempo de consulta (De Almeida, et al., 2012; Parrerias, et al., 2014).

No clareamento caseiro, o custo é mais acessível e o tempo de consulta é reduzido de forma significativa (Auschill, et al., 2005). No entanto, necessita de maior quantidade de sessões para que o resultado final seja satisfatório, um molde preciso da arcada dentária, além de boa instrução de uso e remoção do excesso de gel após a inserção e uma eficaz colaboração do paciente (Bruzell, et al., 2013). Na maioria dos casos, a melhor opção é a combinação de diferentes sistemas de clareamento. Assim sendo, foi sugerida ao paciente essa proposta de associação das técnicas clareadores caseira e de consultório, previamente ao fechamento de diastemas, de forma a otimizar o tempo de trabalho e possibilitar o uso de resinas com colorações mais naturais. Foram realizadas 3 sessões de clareamento de consultório com peróxido de hidrogênio a 35\% (Whiteness HP - FGM. Joinville, SC, Brasil) com barreira gengival (Top Dam - FGM. Joinville, SC, Brasil), com posterior clareamento caseiro utilizando peróxido de carbamida a $16 \%$ por 21 dias.

Nas etapas de fechamento de diastema, o isolamento absoluto é imprescindível, uma vez que é responsável por proporcionar um campo de trabalho com melhor controle da contaminação por saliva e sangue (Benevides, et al., 2019), além de proteger o paciente do risco da ingestão de materiais odontológicos e proporcionar uma melhor visibilidade ao profissional. Uma vez que o campo de trabalho do Cirurgião- Dentista está favorável, há uma elevação na longevidade e qualidade do procedimento executado (Menegaz, 2020). Vale agregar que modificações podem ser efetuadas almejando individualizar cada situação clínica, como é o caso do presente artigo, o qual utilizou o isolamento absoluto modificado, técnica simples e eficaz, para isolamento dos dentes antero-superiores.

Para a execução dessa técnica são inseridos roletes de algodão lateralmente ao freio labial do paciente. No lençol de borracha são realizadas, geralmente, de oito a dez perfurações paralelas, de modo a abranger de pré-molar a pré-molar. Sua fixação pode ser executada com grampos (de número 206 a 209) ou stops, que são fragmentos seccionados do lençol de borracha, 
postos entre os dentes, deixando o lençol estável e passível de controle de fluídos orais, alcançando uma adesão adequada à superfície dentinária (Benevides, et al., 2019). Com esse tipo de isolamento é possível uma boa visualização das margens gengivais, principalmente quando associado ao fio retrator que corrobora também para o controle do exsudato do fluido crevicular (Zeni, et al., 2014).

A aplicação de fio retrator gengival permite o trabalho restaurador em região seca, por meio da retenção de fluidos gengivais e saliva. Também promove melhor visualização do término cervical e margem da restauração (Gouveia, et al., 2017). A manutenção adequada do material restaurador a longo-prazo depende da saúde do tecido periodontal adjacente (Kostic, et al., 2012). Logo, a utilização do fio retrator precisa ser realizada cuidadosamente, a fim de não promover danos ao tecido periodontal no momento de sua inserção (Gupta, et al., 2013). Com esse objetivo, neste trabalho, a aplicação do fio retrator foi feita em passo único, com delicadeza, inibindo possíveis danos às estruturas de suporte e evitando desconforto ao paciente.

Em relação ao condicionamento ácido do esmalte dentário, há décadas é uma técnica bem estabelecida cientificamente e rotineira em procedimentos clínicos restauradores. O esmalte dentário é um tecido de base mineralizada, composto principalmente por cristais de hidroxiapatita, estruturados em prismas. Apresenta $96 \%$ de porção inorgânica (mineral) e apenas $4 \%$ de porção orgânica e água. Devido a essa composição homogênea, a superfície, quando submetida ao condicionamento ácido, a exemplo o ácido fosfórico 37\% (Maquira, Maringá, PR, Brasil), utilizado neste trabalho, cria poros que permitem escoamento de materiais adesivos e, portanto, permite uma retenção micromecânica após polimerização, que estabelece efetividade em adesão na região condicionada (Baratieri, et al.,1995; Ten Cate, et al., 2001; Frankenberger, et al., 2000; Sofan, et al., 2017).

Diferentemente do esmalte, a dentina apresenta composição heterogênea e úmida, representada em $70 \%$ por matéria inorgânica, 20\% de matéria orgânica e 10\% de água (Cecchin, et al., 2008). A dentina superficial apresenta em menor quantidade túbulos dentinários, mas em grande quantidade dentina intertubular, o que dificulta a ação dos ácidos condicionantes, uma vez que o ácido, antes de promover porosidades que capacitariam microinfiltrações com material adesivo, primeiramente age eliminando provável lama dentinária (smear layer), causada pelo preparo do dente, e eliminando dentina intertubular (Cecchin, et al., 2008; Oda, et al., 1999).

Neste trabalho, não houve necessidade de desgastes do órgão dentário para a confecção de restaurações em resina composta, pois o paciente almejava um procedimento conservador e minimamente invasivo, o que foi ao encontro do planejamento do dentista, que notava a presença de espaço de trabalho e possibilidade de adesão de materiais adequada. Logo, a aplicação do condicionamento com ácido fosfórico 37\% (Maquira, Maringá, PR, Brasil) se deu diretamente em esmalte e não houve presença de smear layer ou fatores que prejudicariam a adesão. A etapa de condicionamento ácido aumentou a rugosidade superficial e criou microporosidades, permitindo, assim, a aplicação de sistemas adesivos.

Os sistemas adesivos, por sua vez, apresentam diferentes classificações, relacionadas ao número de passos necessários para a promover a adesão. Podem ser separados por convencionais, necessitando do condicionamento do esmalte e/ou dentina com ácido ácido fosfórico prévio, variando de acordo com o número de passos necessários clinicamente. Também podem se apresentar de formas alternativas, como os sistemas adesivos autocondicionantes, também podendo apresentar diferentes passos para aplicação conforme o produto selecionado, mas que não necessitam de condicionamento com ácido fosfórico prévio por possuir fórmula com monômeros resinosos de $\mathrm{pH}$ ácido, capazes de promoverem micro infiltrações e impregnação por adesivo simultaneamente no substrato dentário. (Inoue, et al., 2003; Carvalho, et al., 2004; Da Cruz, 2021)

O adesivo selecionado para realização do caso clínico foi Adper Single Bond 2 (3M/ESPE, St. Paul, MN, EUA). É um produto adesivo convencional de dois passos, que foi escolhido pelo fácil manuseio e maior efetividade na adesão ao esmalte, se comparado a outras técnicas adesivas alternativas. Possui em sua composição primer e bond no mesmo frasco, necessitando apenas do condicionamento ácido da superfície inicialmente feito. Com a superfície do esmalte do paciente já condicionada, foi 
feito esfregaço com adesivo durante 15 segundos, duas vezes. Após isso foi, enfim, fotopolimerizado por 30 segundos. (Peumans, et al., 2006; Van Meerbeek, et al., 2003; Ergin, et al., 2018)

É válido ressaltar que o contato proximal tem papel relevante no quesito de proteção do periodonto e sua perda pode resultar em inclinações no dente e em injúrias advindas tanto da impactação alimentar, seja ela horizontal ou vertical (Li, et al., 2016), quanto de distúrbios oclusais relacionados ao dente antagonista. Pode ocorrer, ainda o aparecimento de espaços negros advindos da perda da papila interdentária (Paula, 2021), causando, além da debilitação da função, uma debilitação estética. Nesse sentido, para auxiliar na confecção do contato proximal e com a intenção de superar ou contornar tais problemas, foi sugerida a utilização de unimatrix (Unimatrix - TDV. Pomerode, SC, Brasil).

Entre as vantagens que este sistema apresenta ante as matrizes convencionais, cita-se a facilidade de uso e um bom resultado de reanatomização e restabelecimento de ponto de contato (Tateyama, \& Yamamura, 2018), além de ser confortável ao paciente. A facilidade do uso pode ser justificada em detrimento da rigidez da matriz a qual oferece estabilidade e possibilita o estabelecimento do formato do dente já na etapa restauradora, permitindo um correto contorno da superfície proximal, além de tornar a inserção e condensação dos compósitos de resina composta facilitada, impedindo que haja excessos de material restaurador (Paula, 2021).

A resina composta, material restaurador utilizado no caso clínico, detém a vantagem de execução do tratamento de modo pouco invasivo, com menores custos e tempo de espera (Fernandes, et al., 2014), opondo-se às cerâmicas as quais necessitam de maiores desgaste nas estruturas dentárias, além de etapas laboratoriais as quais aumentam a duração e valores do tratamento. (Diegues, et al., 2017). No entanto, quando comparadas a cerâmicas, as resinas perdem na resistência e na maior propensão a pigmentações extrínsecas e, cabe ao paciente, após as apresentações dos materiais, a escolha do procedimento restaurador o qual deseja seguir.

Um dos grandes desafios da técnica incremental de resina composta é a pegajosidade dos compósitos às espátulas de inserção, devido à alta viscosidade dos monômeros que compõem a matriz orgânica (Ribeiro, 2017), o que dificulta a confecção da anatomia dental tão crucial ao procedimento restaurador, podendo gerar falhas no interior da restauração (Munchow, et al., 2016), influenciando de forma direta em sua qualidade, longevidade e desempenho clínico. Dessa maneira, os dentistas têm buscado métodos auxiliares para facilitar o manuseio clínico dos incrementos de resina composta (Cacique, 2019). Como exemplo, tem-se a utilização de resinas fluidas denominadas de líquido modelador.

O uso do modelador de resinas melhora a inserção e a escultura dentária, corroborando para um resultado final satisfatório (Cacique, 2019). No entanto, há estudos que afirmam que a utilização desses agentes pode afetar o grau de conversão e a densidade de ligações cruzadas de nanocompósitos (Paula, et al., 2016). Uma deficiência no grau de conversão afeta algumas propriedades das resinas (Ribeiro, 2017) como dureza, resistência à compressão, resistência à flexão e às tensões, solubilidade e reações de degradação e estabilidade dimensional e de cor.

Tendo conhecimento a respeito desse fato supracitado, o uso do agente umectante de compósitos se restringiu apenas à última camada de resina de esmalte de modo a entregar uma boa anatomia dental, evitando a presença de falhas (Munchow, et al., 2016) e rugosidades na superfície da restauração sem que houvesse um comprometimento significativo ao grau de conversão. Ademais, após a fotopolimerização da última camada do modelador de resinas (BISCO, Illinois, Schaumburg) foi aplicado gel hidrossolúvel de modo a isolar a resina do oxigênio, do ambiente, objetivando uma polimerização completa e consequente melhora nas propriedades físico-mecânicas da restauração (Queiroz, 2019).

A aplicação do gel hidrossolúvel está diretamente ligada ao grau de conversão de monômeros, concorrendo com um aumento da quantidade de polímeros na camada superficial (Koga, et al., 2011). Tal processo se justifica além do fato de o oxigênio presente no ambiente reagir com os radicais livres da camada superficial de resina composta, com o fato de que a aplicação do gel associada à polimerização adicional (Queiroz, 2019) pode gerar maior dose de energia que aumenta o grau de 
conversão da resina composta (Calheiros, et al., 2004; Halvorson, et al., 2002; Emami, et al., 2003) e influência de forma direta na redução da rugosidade superficial dos espécimes (Korkmaz, et al., 2008).

Por fim, como última etapa dos tratamentos restauradores, tem-se o acabamento e polimento, que previnem o acúmulo de restos alimentares os quais podem vir a gerar maior pigmentação nas resinas compostas. Além de aumentar a lisura e longevidade das restaurações, com o acabamento é possível melhorar a anatomia dental desejada e com o polimento, as rugosidades presentes são reduzidas e o brilho da restauração é realçado (Silva \& Oliveira Nunes, 2019). No caso relatado, o acabamento e polimento das restaurações proporcionou ao paciente a individualização anatômica que o mesmo desejava, gerando grande satisfação, devolvendo sua autoestima ao final do tratamento.

\section{Conclusão}

A resina composta se mostrou uma excelente alternativa quando utilizada de maneira correta por um profissional capacitado, que compreenda os princípios estéticos e psicológicos de seu paciente. A utilização de diferentes métodos de trabalho associados proporcionou o sucesso do tratamento restaurador citado e foram suficientes para superar as expectativas do paciente, devolvendo ao mesmo a estética de seus dentes, elevando sua autoestima. Ademais, é válido ressaltar que o uso da resina composta combinada ao agente umectante na última camada de esmalte permitiu uma escultura final com menor quantidade de bolhas e interfaces de incremento, facilitando o acabamento e polimento final. Entretanto, é de extrema importância entender como o uso de agentes modeladores nas restaurações pode influenciar nas propriedades físicas de materiais resinosos, uma vez que a qualidade desses parâmetros implica na caracterização final da superfície da restauração.

Diante do que foi exposto neste relato de caso, sugere- se a realização de novas pesquisas que acompanhem 0 desempenho clínico do procedimento a longo prazo. Além disso, pesquisas que analisem os fatores que interferem nessa longevidade das restaurações estéticas em resina direta também são relevantes e necessárias.

\section{Referências}

Ahmad, I. (2005). Anterior dental aesthetics: Dental perspective. British dental journal, 199(3), 135-141.

Arndt, E. M., Travis, F., Lefebvre, A., Niec, A., \& Munro, I. R. (1986). Beauty and the eye of the beholder: Social consequences and personal adjustments for facial patients. British journal of plastic surgery, 39(1), 81-84.

Auschill, T, Hellwig, E, Schmidale, S, Sculean, A, Arweiler, N.B. (2005). Efficacy, side-effects and patients' acceptance of different bleaching techniques. Oper Dent, 30:156-163.

Baratieri, L. N. et al. (1995) Estética - Restaurações Adesivas Diretas Em Dentes Anteriores Fraturados. São Paulo: Ed. Santos. 6: $135-205$.

Barbosa, D. C., De’Stefani, T. P. Ceretta, L. B., Ceretta, R. A. Simões, P. W., \& D’Altoé, L. F. (2017). Estudo comparativo entre as técnicas de clareamento dental em consultório e clareamento dental caseiro supervisionado em dentes vitais: uma revisão de literatura. Revista de Odontologia da Universidade Cidade de São Paulo, 27(3), 244-252.

Bayraktar, E. T., Atali, P. Y., Korkut, B., Kesimli, E. G., Tarcin, B., \& Turkmen, C. (2021). Effect of Modeling Resins on Microhardness of Resin Composites. European journal of dentistry, 15(3), 481-487. https://doi.org/10.1055/s-0041-1725577

Benevides, A. A. A., Venâncio, A. E. F., \& Feitosa, V. P. (2019). A influência do isolamento absoluto no sucesso de restaurações diretas e tratamento endodôntico: uma revisão de literatura. Revista Odontológica de Araçatuba, 35-40.

Bruzell, E.M, Pallesen, U, Thoresen, N.R et al. (2013). Side effects of external tooth bleaching: a multi-centre practice-based prospective study. Br Dent J., 215:1-8.

Cacique, A. O. A. (2019). Efeito do uso de líquido modelador na estabilidade de cor e translucidez de resina composta. Campos GV, 3-20.

Calamia, V., \& Pantzis, A. (2015). Simple case treatment planning: diastema closure. Dent Clin North Am, 59(3), 655-64.

Calheiros, F.C, Braga, R.R, Kawano, Y, Ballester, R.Y.(2004). Relationship between contraction stress and degree of conversion in restorative composites. Dent Mater ,20(10):939-46.

Calixto, L. R., Coelho Bandeca, M., \& Ferrarezi De Andrade, M. (2011). Enceramento diagnóstico: previsibilidade no tratamento estético indireto. Revista Dental Press de Estética, 8(4). 
Canuto, M. S. B., Assis, R. S., Gouveia, P. M. P., \& Nemr, K. (2006). Análise comparativa entre presença de diastemas e tipos faciais. Revista CEFAC, 8(2), $162-170$.

Carvalho, R. M. D., Carrilho, M. D. O. Pereira, L. C. G., Garcia, F. C. P., Marquezini Jr., L., Silva, S. M. A., \& Kussmaul, A. P. M. (2004). Sistemas adesivos: fundamentos para aplicação clínica. Biodonto, 2(1), 1-89.

Cecchin, D., Farina, A. P., Spazzin, A. O., Galafassi, D., Barbizam, J. V. B., \& Carlini-Júnior, B. (2008). Influência da profundidade dentinária na resistência à microtração de sistemas adesivos de condicionamento ácido total e autocondicionante. Revista Odonto Ciência, 23(2).

Chu, C. H., Zhang, C. F., \& Jin, L. J. (2011). Treating a maxillary midline diastema in adult patients: a general dentist's perspective. The Journal of the American Dental Association, 142(11), 1258-1264.

Cruz, L. S., Oliveira, G. M. (2021). Morphological Analysis Of Enamel And Dentin After The Use Of Two Types Of Adhesive Systems. Rev Fac Odontol Univ Fed Bahia ,51(1): 19-28.

da Costa, J. B., McPharlin, R., Paravina, R. D., \& Ferracane, J. L. (2010). Comparison of at-home and in-office tooth whitening using a novel shade guide. Operative dentistry, 35(4), 381-388.

De Almeida, L. C. A. G., Costa, C. A. S, Riehl H, dos Santos P, Sundfeld, R. H, Briso, A. L. (2012). Occurrence of sensitivity during at-home and in-office tooth bleaching therapies with or without use of light sources. Acta Odontol Latinoam, 25:3-8.

Diegues, M. A., Marques, E., Miyamotto, P. A. R., \& Penteado, M. M. (2017). Cerâmica X Resina Composta: o que utilizar? Revista Uningá, 51(1).

Emami, N, Soderholm, K. J., Berglund, L. A. (2003). Effect of light power density variations on bulk curing properties of dental composites. J Dent, 31(3):18996.

Ergin, E., Kutuk, Z. B., Cakir, F. Y., \& Gurgan, S. (2018). Comparison of two different composite resins used for tooth reshaping and diastema closure in a 4year follow-up. Nigerian journal of clinical practice, 21(9), 1098-1106.

Estrela, C. (2018). Metodologia Científica: Ciência, Ensino, Pesquisa. Editora Artes Médicas.

Felix, B. M. D. M., Oliveira, L. S. D., \& Gonçalves, T. D. A. (2019). Análise comparativa entre técnicas e géis clareadores dentais de diferentes concentrações: revisão de literatura.

Fernandes, H. K., Silva, R., Marinho, M. A. S., de Souza Oliveira, P. O. Ribeiro, J. C. R., \& Moyses, M. R. (2014). Evolução da resina composta: revisão da literatura. Revista da universidade vale do rio verde, 12(2), 401-411.

Ferracane, J. L. (2011). Resin composite—state of the art. Dental Materials, 27(1), 29-38. https://doi.org/10.1016/j.dental.2010.10.020

Frankenberger R, Krämer N, Petschelt (2000). Long-term effect of dentin primers on enamel bond strength and marginal adaptation. Oper Dent. 25(1), 11-9.

Frese, C., Schiller, P., Staehle, H. J., \& Wolff, D. (2013). Recontouring teeth and Closing Diastemas with direct composite buildups: A 5-YEAR follow-up. Journal of Dentistry, 41(11), 979-985. https://doi.org/10.1016/j.jdent.2013.08.009

Gupta, A., Prithviraj, D. R., Gupta, D., \& Shruti, D. P. (2012). Clinical evaluation of three new gingival retraction systems: A research report. The Journal of Indian Prosthodontic Society, 13(1), 36-42. https://doi.org/10.1007/s13191-012-0140-y

Halvorson, R.H, Erickson, R.L, Davidson, C.L. (2002). Energy dependent polymerization of resin-based composite. Dent Mater,18(6):463-9.

Haywood, V.B. (2006). Number of in-office light-activated bleaching treatments needed to achieve patient satisfaction. Quintessence Int, 37:115-120.

Inoue, S., Vargas, M. A., Abe, Y., Yoshida, Y., Lambrechts, P., Vanherle, G., \& Van Meerbeek, B. (2003). Microtensile bond strength of eleven contemporary adhesives to enamel. American Journal of Dentistry, 16(5), 329-334.

Kabbach, W., Sampaio, C. S., \& Hirata, R. (2018). Diastema closures: A novel technique to ensure dental proportion. Journal of Esthetic and Restorative Dentistry, 30(4), 275-280. https://doi.org/10.1111/jerd.12397

Karadas, M., \& Duymus, Z. Y. (2015). In vitro evaluation of the efficacy of different over-the-counter products on tooth whitening. Brazilian dental journal, $26,373-377$.

Keene, H. J. (1963). Distribution of diastemas in the dentition of man. American Journal of Physical Anthropology, 21(4), 437-441. https://doi.org/10.1002/ajpa.1330210402

Koga, K., Tsujimoto, A., Ishii, R., Iino, M., Kotaku, M., Takamizawa, T., \& Miyazaki, M. (2011). Influence of oxygen inhibition on the surface free-energy and dentin bond strength of self-etch adhesives. European journal of oral sciences, 119(5), 395-400.

Korkmaz, Y, Ozel, E, Attar, N, Aksoy, G. (2008) The influence of one-step polishing systems on the surface roughness and microhardness of nanocomposites. Operative dentistry,33(1), 44-50.

Kossatz, S., Dalanhol, A. P., Cunha, T., Loguercio, A., \& Reis, A. (2011). Effect of light activation on tooth sensitivity after in-office bleaching. Operative dentistry, 36(3), 251-257.

Kostić, I., Najman, S., Kostić, M., \& Stojanović, S. (2012). Comparative review of gingival retraction agents. Acta Medica Medianae, 81-84. https://doi.org/10.5633/amm.2012.0114 
Lempel, E., Lovász, B. V., Meszarics, R., Jeges, S., Tóth, Á., \& Szalma, J. (2017). Direct resin Composite restorations for Fractured maxillary teeth and diastema closure: A 7 years Retrospective evaluation of survival and influencing factors. Dental Materials, 33(4), 467-476. https://doi.org/10.1016/j.dental.2017.02.001

Li, Q. L., Ying Cao, C., Xu, Q. J., Xu, X. H., \& Yin, J. L. (2016). Atraumatic restoration of vertical food impaction with an open contact using flowable composite resin aided by cerclage wire under tension. Scientifica, 2016.

Lima, T. C. D. C. (2019). Redução de diastema utilizando resina composta direta: relato de caso.

Lynch, C. D., Frazier, K. B., McConnell, R. J., Blum, I. R., \& Wilson, N. H. F. (2011). Minimally invasive management of dental caries. The Journal of the American Dental Association, 142(6), 612-620. https://doi.org/10.14219/jada.archive.2011.0243

Marques, F. P. (2019). O impacto social do diastema mediano superior.

Menegaz, Z. A. (2020). Uso do Isolamento Absoluto em Odontologia. Odontologia-Tubarão.

Michelon, C., Hwas, A., de Freitas Borges, M., da Costa Marchiori, J., \& Susin, A. H. (2009). Restaurações diretas de resina composta em dentes posterioresconsiderações atuais e aplicação clínica. Revista da Faculdade de Odontologia-UPF, 14(3).

Migliau, G. (2017). Classification review of Dental Adhesive systems: From the IV generation to the universal type. Annali Di Stomatologia, 8(1), 1. https://doi.org/10.11138/ads/2017.8.1.001

Mondelli, J. (2017). Fundamentos da dentística operatória. Grupo Gen-Livraria Santos Editora.

Mounika, A., Mandava, J., Roopesh, B., \& Karri, G. (2018). Clinical evaluation of color change and tooth sensitivity with in-office and home bleaching treatments. Indian Journal of Dental Research, 29(4), 423.

Munchow, E.A, Sedrez-Porto, J.A, Piva. E, Pereira-Cenci, T, Cenci, M.S. (2016). Use of dental adhesives as modeler liquid of resin composites. Dent Mater, 32(4):570-7.

Nunes Gouveia, T. H. Dias Theobaldo, J., Vieira-Junior, W., Alves Nunes Leite Lima, D., \& Baggio Aguiar, F. H. (2017). Esthetic smile rehabilitation of anterior teeth by treatment with biomimetic restorative materials: A case report. Clinical, Cosmetic and Investigational Dentistry, Volume 9, 27-31. https://doi.org/10.2147/ccide.s130698

Oda, M., Matos, A. B., \& Liberti, E. A. (1999). Morfologia da dentina tratada com substâncias dessensibilizantes: avaliação através da microscopia eletrônica de varredura. Revista de Odontologia da Universidade de São Paulo, 13, 337-342.

Parreiras, S, Vianna, P, Kossatz, S, Loguercio, A. D, Reis, A. (2014). Effects of light activated in-office bleaching on permeability, microhardness, and mineral content of enamel. Oper Dent, 39:E225-E230

Paula, F. C, Valentin, R. S, Borges, B. C, Medeiros, M. C, Oliveira, R. F, \& Silva, A. O. (2016). Effect of instrument lubricants on the surface degree of conversion and crosslinking density of nanocomposites. J Esthet Restor Dent. 28(2), 85-91.

Paula, R.S.N. (2021). Sistemas de matrizes e cunhas para restabelecer o contato proximal em restaurações classe II compostas e complexas. Universidade De Uberaba, 5-22.

Perozzo, F., Rodrigues, j. F., \& Felizardo, k. R. (2017). Produtos clareadores "over-the-counter"(etc): revisão de literatura. Revista uningá review, 29(3).

Peumans, M., Kanumilli, P., De Munck, J., Van Landuyt, K., Lambrechts, P., \& Van Meerbeek, B. (2006). Clinical effectiveness of contemporary adhesives: A systematic review of current clinical trials. The Journal of Prosthetic Dentistry, 95(6), 472. https://doi.org/10.1016/j.prosdent.2006.02.019

Queiroz, L. L. (2019). Influência do gel hidrossolúvel e diferentes sistemas abrasivos no acabamento e polimento de uma resina composta nanohíbrida: simulação de superfície interproximal. Universidade Federal De Uberlândia.

Ribeiro, L. S. (2017) Efeito de uma resina modeladora sobre o grau de conversão de um compósito nanoparticulado. Universidade Federal Do Rio Grande Do Norte. 4-22

Ricardo Barros de Campos, P., Rocha Maia, R., Rodrigues de Menezes, L., Ferreira Barbosa, I., Carneiro da Cunha, A., \& Damiana da Silveira Pereira, G. (2017). Isolamento absoluto - O Segredo para o sucesso Na Técnica DE Fechamento de Diastema Com resinas compostas. The international journal of esthetic dentistry, 02(04), 682. https://doi.org/10.20432/ijed682

Rodríguez-Martínez, J., Valiente, M., \& Sánchez-Martín, M. J. (2019). Tooth whitening: From the established treatments to novel approaches to prevent side effects. Journal of Esthetic and Restorative Dentistry, 31(5), 431-440.

Román-Rodríguez, J. L. et al. (2020). A tooth whitening and chemical abrasive protocol for the treatment of developmental enamel defects. The Journal of prosthetic dentistry, 123(3), 379-383.

Romero, M. F., Babb, C. S., Brenes, C., \& Haddock, F. J. (2018). A multidisciplinary approach to the management of a maxillary midline diastema: A clinical report. The Journal of Prosthetic Dentistry, 119(4), 502-505. https://doi.org/10.1016/j.prosdent.2017.06.017

Sedrez-porto, J. A., Münchow, E. A., Brondani, L. P., Cenci, M. S. Pereira-Cenci, T. (2016). Effects of modeling liquid/resin and polishing on the color change of resin composite. Brazilian Oral Research, 30(1). https://doi.org/10.1590/1807-3107bor-2016.vol30.0088

Sofan, E., Sofan, A., Palaia, G., Tenore, G., Romeo, U., \& Migliau, G. (2017). Classification review of dental adhesive systems: from the IV generation to the universal type. Annali di stomatologia, 8(1), 1.

Sundfeld, D., Pavani, C. C., Schott, T. C., Machado, L. S., Pini, N. I. P., de Magalhães Bertoz, A. P., \& Sundfeld, R. H. (2019). Dental bleaching on teeth submitted to enamel microabrasion 30 years ago - a case report of patients' compliance during bleaching treatment. Clinical oral investigations, 23(1), 321-326. 
Research, Society and Development, v. 10, n. 12, e349101220635, 2021

(CC BY 4.0) | ISSN 2525-3409 | DOI: http://dx.doi.org/10.33448/rsd-v10i12.20635

Tateyama, A., \& Yamamura, S. (2018). Restauração de Classe II em resina composta da anatomia ao ponto de contato ideais. Full dent. sci, $127-131$.

Tay, L. Y., Kose, C., Loguercio, A. D., \& Reis, A. (2009). Assessing the effect of a desensitizing agent used before in-office tooth bleaching. The Journal of the American Dental Association, 140(10), 1245-1251.

Ten Cate R. (2001) Histologia Bucal - desenvolvimento, estrutura e função. In:Estrutura do Esmalte. Editora Guanabara. 5, $205-221$.

Van Meerbeek, B., De Munck, J., Yoshida, Y., Inoue, S., Vargas, M., Vijay, P., \& Vanherle, G. (2003). Adhesion to enamel and dentin: current status and future challenges. Operative Dentistry-University Of Washington-, 28(3), 215-235.

Zeni, F., Ilkiu, R. E., Schmitt, J., \& Martarello, C. (2014). Isolamento absoluto modificado: alternativa para trabalhos em dentes anteriores. Ação Odonto, 2(3), $18-18$. 